\title{
Scanning electron microscopy assessment of the load-bearing capacity of cad/cam-fabricated molar crowns
}

\author{
Emine Göncü BAŞARAN(a) \\ Güliz AKTAŞ(b) \\ Pekka VALLITTU(c) iD \\ Lippo LASSILA(c) \\ Mehmet Cudi TUNCER(d) \\ (a) University of Dicle, Faculty of Dentistry, \\ Department of Prosthetic Dentistry, \\ Diyarbakır, Turkey. \\ (b) University of Hacettepe, Faculty of \\ Dentistry, Department of Prosthetic \\ Dentistry Ankara, Turkey. \\ (c) University of Turku, Institute of Dentistry, \\ Department of Biomaterials Science, \\ Turku, Finland. \\ (d) Dicle University, Faculty of Medical Science, \\ Department of Anatomy, Diyarbakır, Turkey.
}

Declaration of Interests: The authors certify that they have no commercial or associative interest that represents a conflict of interest in connection with the manuscript.

Corresponding Author:

MehmetCudi Tuncer

drcudi@hotmail.com

https://doi.org/10.1590/1807-3107bor-2020.vol34.0035

Submitted: July 31, 2019

Accepted for publication: January 6, 2020

Last revision: March 12, 2020

\begin{abstract}
Although fiber-reinforced composites are commonly used in dental practice, whether fiber-reinforced crowns and fixed partial dentures can be used as definitive prostheses remains to be determined. This study used scanning electron microscopy to evaluate the load-bearing capacity of non-reinforced and fiber-reinforced composite (FRC) molar crowns prepared by computer-aided design/computer-aided manufacturing (CAD/CAM). The crowns were fabricated from three empirical FRC blocks, one empirical composite block, and one commercial ceramic block. The FRC resin was prepared by mixing $\mathrm{BaO}$ silicate particles, E-glass fiber, and dimethacrylate resin. Specimens were divided into five groups $(n=10)$, differing in the amounts of filler, resin, and fiber. Crowns were statically loaded until fracture. One-way analysis of variance and Tukey's post hoc multiple comparison tests were used for statistical analyses. The groups showed significant differences in load-bearing capacity; empirical bidirectional FRC resin blocks had the highest capacity, while commercial ceramic blocks had the lowest capacity. Molar crowns formed from FRC resin blocks had higher load-bearing capacity compared to non-reinforced composite resin and ceramic blocks. These results show that fiber reinforcement increased the load-bearing capacity of molar crowns.
\end{abstract}

Keywords: Molar; Tooth Crown; Microscopy, Electron, Scanning; Computer-Aided Design; Humans

\section{Introduction}

Due to higher expectations regarding esthetic outcomes, tooth-colored and metal-free restorations have been introduced. All-ceramic crowns are preferred due to their outstanding esthetic outcomes, biocompatibility, and durability. ${ }^{1,2}$ However, abrasive wear of the opposing natural teeth and the high cost of all-ceramic crowns limit their use. Moreover, the failure rate due to brittle catastrophic fracture is relatively high.,4

In recent decades, the use of composite resin in posterior teeth has increased, ${ }^{5,6}$ particularly with the development of reinforced composite resins. Fiber-reinforced composites (FRCs) have been used for replacement of missing teeth and conventional dental restorations. ${ }^{78,9}$ The advantages of FRCs include high translucency, high bonding strength, and ease of repair. ${ }^{10,11}$ 
Composites are isotropic materials showing similar properties in all directions. The mechanical behavior of FRCs differs from that of particulate filler composites. The characteristics of FRCs vary from isotropic to anisotropic. The direction, volume, and location of the fibers, as well as fiber orientation, adhesion, and water sorption of the resin matrix, influence the mechanical properties of FRCs. ${ }^{12,13}$ Multidirectional reinforcement minimizes the anisotropic behavior of unidirectional fibers, but strength is reduced. ${ }^{12}$ When fibers are equal to or longer than the critical fiber length $(0.5-1.6 \mathrm{~mm})$, stress is transmitted between the fibers and the polymer matrix. ${ }^{14,15,16}$ Although much is known about the properties of FRCs, there is relatively little information available on the properties of FRCs when combined with particulate filler composite. ${ }^{17}$

Within a structure, fibers can be unidirectional, bidirectional, braided, or woven. ${ }^{18}$ Unidirectional fibers exhibit a similar diameter, have a circular cross-section, and are straight, tightly packed, and anisotropic; they also show a reinforcing effect when forces are applied perpendicularly to the direction of the fibers. However, bidirectional fibers exhibit an orthotropic reinforcing effect, i.e., they exert reinforcing effects in two directions, and thus improve structural strength. ${ }^{18}$

Discontinuous FRCs have been improved in terms of toughness. ${ }^{18}$ The behaviors of randomly oriented discontinuous fibers are affected by the fiber volume fraction. If fibers are randomly and homogenously oriented, they show isotropic properties; however, if they have sufficient length, they demonstrate anisotropic properties similar to those of long fibers. ${ }^{19}$

Several materials can be used to fabricate crowns via computer-aided design/computer-aided manufacturing (CAD/CAM), including IPS e.max CAD blocks (Ivoclar Vivadent AG, Schaan, Liechtenstein). IPS e.max CAD is an esthetic lithium disilicate glass ceramic that can be used for efficient fabrication of highly esthetic restorations. Most restorations can be produced with IPS e.max CAD blocks, including veneers and three-unit bridges. Although there have been many studies on FRC fixed partial dentures, few have been performed on FRC crowns. A variety of materials can be used for restoring posterior crowns, but controversy surrounding the ideal material still remains.
In the present study, we used scanning electron microscopy (SEM) to evaluate the load-bearing capacity of molar crowns fabricated using a CAD/CAM milling system, as well as bonding and impregnation of different fiber-filler-resin combinations. Specifically, we compared impregnated, bidirectional, randomly oriented, and unidirectionally oriented FRC resin blocks to a non-reinforced composite block and to a commercially available prefabricated ceramic block. We hypothesized that FRC blocks would exhibit higher load-bearing capacity than the other blocks.

\section{Methods}

\section{Preparation}

Fifty crowns were fabricated in the laboratory; the specimens were divided into five groups $(n=10)$, i.e., three FRCs, one non-reinforced composite, and one commercial ceramic block. A zirconia crown (ICE Zirconia; Zirkonzahn, Bruneck, Italy) was fabricated, simulating the first upper molar, using a manual milling machine (Zirkonzahn). Crowns were made using 1.5-mm axial shoulder reductions in accordance with the cementoenamel junction. Axial reduction was measured from the preparation margins. Occlusal reduction $(2 \mathrm{~mm})$ was then performed.

The molar crowns were fabricated using five materials. Empirical continuous unidirectional fiber-reinforced composite (EUFRC) was prepared by mixing $35 \mathrm{wt} \%$ barium silicate $\left(\mathrm{BaOSiO}_{2}\right.$ filler particles ( $1 \%$ silane, $0.7-\mu \mathrm{m}$ glass particle size) with $35 \mathrm{wt} \%$ dimethacrylate-polymethyl methacrylate resin matrix. Mixing was performed using a high-speed centrifuge for 5 minutes at 3,500 rpm (SpeedMixer DAC; Hauschild Engineering, Hamm, Germany); the mixing procedure was the same in all groups. Because of the thin and long structure of the unidirectional E-glass fiber, the mixture was impregnated with $30 \mathrm{wt} \%$ fiber, of the same length as the commercial ceramic block, to achieve a homogenous structure. Empirical continuous bidirectional fiber-reinforced composite (EBFRC) was prepared by mixing $38 \mathrm{wt} \%$ barium silicate filler particles with $38 \mathrm{wt} \%$ dimethacrylate-polymethyl methacrylate resin matrix. Bidirectional E-glass fiber was prepared with dimensions identical to those of the 
commercial ceramic block. The resin composite was impregnated with $24 \mathrm{wt} \%$ bidirectional E-glass fiber to achieve a homogenous structure. Empirical random fiber-reinforced composite (ERFRC) was prepared by mixing $28.5 \mathrm{wt} \%$ E-glass fiber (length $=10 \mathrm{~mm}$ ) with $26.5 \mathrm{wt} \%$ dimethacrylate-polymethyl methacrylate resin matrix, which yielded wet fiber surfaces. Then, $45 \mathrm{wt} \%$ barium silicate filler particles ( $1 \%$ silane, $0.7-\mu \mathrm{m}$ glass particle size) were added gradually to create the final homogenous structure. Empirical composite (EC) was prepared by mixing $25.6 \mathrm{wt} \%$ dimethacrylate-polymethyl methacrylate resin matrix with $74.3 \mathrm{wt} \%$ barium silicate filler particles ( $1 \%$ silane, $0.7-\mu \mathrm{m}$ glass particle size). Fillers were added gradually to achieve the final weight fraction. Finally, a prefabricated commercial ceramic block (CCB) (IPS e.max) was used as a control.

Fibers were used in the reinforced groups in various ratios. Fibers were impregnated with the manually prepared resin mixture and then placed in the mold. Because of the difference in structure among fibers, the amount of impregnated resin used varied. A silicon mold was used to fabricate blocks from FRCs and EC, with dimensions identical to those of the CCB. The FRCs and EC were placed in the mold and vacuum was applied for 1 hour; heat curing was then conducted under high pressure using an Ivomat (Ivoclar AC, Schaan, Liechtenstein) polymerization device.

Photographs of the zirconia model obtained using an intraoral camera were exported to the Cerec 3 Sirona CAD-CAM system (Sirona Cerec MC L; Sirona Dental Systems, Bensheim, Germany). The CCB, EC, and FRC blocks were milled using Cerec 3 Sirona. The CAD design was identical for all crowns.

\section{Test procedure}

Molar crowns were cemented to the zirconia model using a dual curing-luting agent (Rely X Unicem App; 3M ESPE AG, Seefeld, Germany) and finger pressure, in accordance with the manufacturer's protocol. The pre-cementation procedure was as follows: lithium disilicate crowns were etched with $5 \%$ hydrofluoric acid (etching gel: Ivoclar Vivadent AG) for 20 seconds, washed with an air/water spray for 20 seconds, and coated with silane (RelyX Ceramic Primer; 3M
ESPE AG). As pre-treatment was applied to ensure mechanical microretention in the zirconia; specimens were sandblasted using an airborne particle abrasion technique with $50-\mu \mathrm{m}$ alumina particles.

Following cementation, each specimen was placed in distilled water at ambient temperature for at least 24 hours. The molar crown models were placed in the testing device. The load-bearing capacity of each molar crown was evaluated using a universal testing machine (Model LRX; Lloyd Instruments Ltd., Fareham, UK) at a cross-head speed of $1.0 \mathrm{~mm} /$ minute; data were recorded using Nexygen software (Lloyd Instruments Ltd.). A load was applied to the central fossa of the pontic using a steel ball (Ø3 $\mathrm{mm})$. The loading event was recorded until final fracture occurred. Initial failure (IF) and final failure (FF) were determined using the method of Dyer et al. ${ }^{12}$ Following the loading test, the specimens were assessed in terms of failure mode: catastrophic fracture, partial fracture, or delamination.

\section{Scanning electron microscopy (SEM)}

Surfaces examined by SEM were abraded using $600-, 800-, 1,000-$, or 1,200-grit abrasives. Subsequently, the surfaces were polished with their own polishing felts (Streuers, Copenhagen, Denmark) using 6-, 3-, 1-, or 1/4- $\mu \mathrm{m}$ diamond polishing paste. The samples were placed in an ultrasonic cleaner (USG 4000 Ultraschall; Dentaurum, Ispringen, Germany) for 10 minutes after each polishing paste application. Then, $10 \%$ phosphoric acid and $5 \% \mathrm{NaOCl}$ were applied for 10 minutes. All samples were rinsed in distilled water for 1 minute and then dried. The prepared surfaces were coated with gold film in an airless environment. Analyses were performed with a scanning electron microscope at 750-1,000× magnification.

\section{Statistical analysis}

Statistical analysis was performed using SPSS for Windows (version 21.0; IBM Corp., Armonk, USA). Results are shown as means and ranges with standard deviation or standard error. One-way analysis of variance and Tukey's multiple comparison post hoc tests were used for comparison of qualitative variables among groups. A p-value $<0.01$ was considered statistically significant. 


\section{Results}

\section{Test findings}

The data on the load-bearing capacity of the crowns are shown in Table. Statistical analysis was performed on the FF values. One-way analysis of variance indicated that the groups differed significantly in load-bearing capacity (df: 4, F = 19.1). Tukey's tests were performed to evaluate differences among groups (Table). EBFRC showed the highest mean load-bearing capacity. However, there was no significant difference between EBFRC and ERFRC, or between EBFRC and EUFRC (both $p>0.05$ ). Although CCB exhibited the lowest load-bearing capacity, there was no significant difference between EC and CCB ( $p>0.05)$. The difference between EBFRC and EC was statistically significant $(p<0.01)$, as was that between EUFRC and $C C B$, EBFRC and $C C B, E R F R C$ and $C C B$, and $\mathrm{EC}$ and CCB (all $\mathrm{p}<0.001)$.

\section{Scanning electron microscopy findings}

The SEM images demonstrated that FRC blocks showed satisfactory impregnation with the resin; there were no pores in the polymer matrix (Figure A-C). The EC block had a homogenous structure (Figure D). Different fiber ratios and fiber orientations among the groups were also shown by the SEM images.

\section{Discussion}

The results of this study supported our hypothesis that load-bearing capacity is significantly higher for FRC blocks than for composite resin and ceramic blocks. A previous study determined that dental restorations in the molar area should withstand a weight of $\leq 500 \mathrm{~N}$, with a likely additional load of $\leq 200 \mathrm{~N} .^{20}$ Another study indicated that FRC crowns could bear loads of $>1,000 \mathrm{~N} .{ }^{21}$ In the present study, the load-bearing capacity of our FRC and composite crowns was significantly higher than that reported in most previous studies. ${ }^{22-24}$ This high fracture strength appears to be sufficient for withstanding the occlusal force applied by natural dentition. ${ }^{21}$ The cumulative success rate of FRC molar crowns after 36 months was estimated at $82 \%{ }^{25}$ In another clinical study, FRC and ceramic restorations showed similar survival rates over a 4-year follow-up period. ${ }^{26}$ The results of the present study show that fiber reinforcement can promote both survival and success.

Ceramic and composite resin crowns have a limited capacity to reduce deformation stress at a crack tip. ${ }^{27}$ The high brittleness and low fracture toughness of composites prevent their use in large tensile restorations. ${ }^{28}$ Composites typically show local failure in two or more pieces before rupture. ${ }^{29}$ Fiber reinforcement can slow down or attenuate crack propagation by supporting the composite resin layer, thereby reducing the incidence of catastrophic and instantaneous failures. ${ }^{12}$ Reinforced composite resin is easier to repair when a crack or rupture occurs. A previous study demonstrated that, under axial loading, lithium disilicate and multiphase resin composites exhibited similar fracture strength. ${ }^{30}$ Another study showed that the strength of a full ceramic system with lithium disilicate fracture resistance was lower than that of fiber-reinforced indirect composites, ${ }^{31}$ the results of the present study were similar.

Although many studies have been concerned with FRC restorations, there is no clear consensus

Table. Descriptive statistics for groups and multiple comparison of final failure with real P values for all groups according to ANOVA.

\begin{tabular}{|c|c|c|c|c|c|c|c|c|c|c|}
\hline \multirow{2}{*}{ Groups } & \multirow{2}{*}{$\mathrm{n}$} & \multirow{2}{*}{ Mean } & \multirow{2}{*}{$\mathrm{Sd}$} & \multirow{2}{*}{$\mathrm{Se}$} & \multirow{2}{*}{ Maximum } & \multirow{2}{*}{ Minimum } & \multirow{2}{*}{$\mathrm{F}$} & \multicolumn{3}{|c|}{ Multiple Comparison Differences Groups } \\
\hline & & & & & & & & $* * *$ & * & n.s. \\
\hline EUFRC (1) & 8 & 1284.5 & 207.5 & 73.6 & 1682.0 & 1038.0 & & & \multirow{5}{*}{$2-4$} & $1-2$ \\
\hline EBFRC (2) & 8 & 1437.1 & 92.2 & 36.2 & 1596.0 & 1325.0 & & $1-5$ & & $1-3$ \\
\hline ERFRC (3) & 8 & 1288.7 & 239.9 & 84.8 & 1671.0 & 1033.0 & 19.124 & $2-5$ & & $1-4$ \\
\hline $\mathrm{EC}(4)$ & 8 & 1024.1 & 113.8 & 40.2 & 1220.0 & 980.0 & & $3-5$ & & $2-3$ \\
\hline CCB (5) & 8 & 910.7 & 83.1 & 29.4 & 996.0 & 868.0 & & $4-5$ & & $3-4$ \\
\hline
\end{tabular}

Visual inspection revealed three different fracture types: catastrophic fractures were observed in EC and CCB samples; partial fractures in EC, EUFRC, and ERFRC samples; and delamination in EUFRC, EBFRC, and ERFRC samples. 

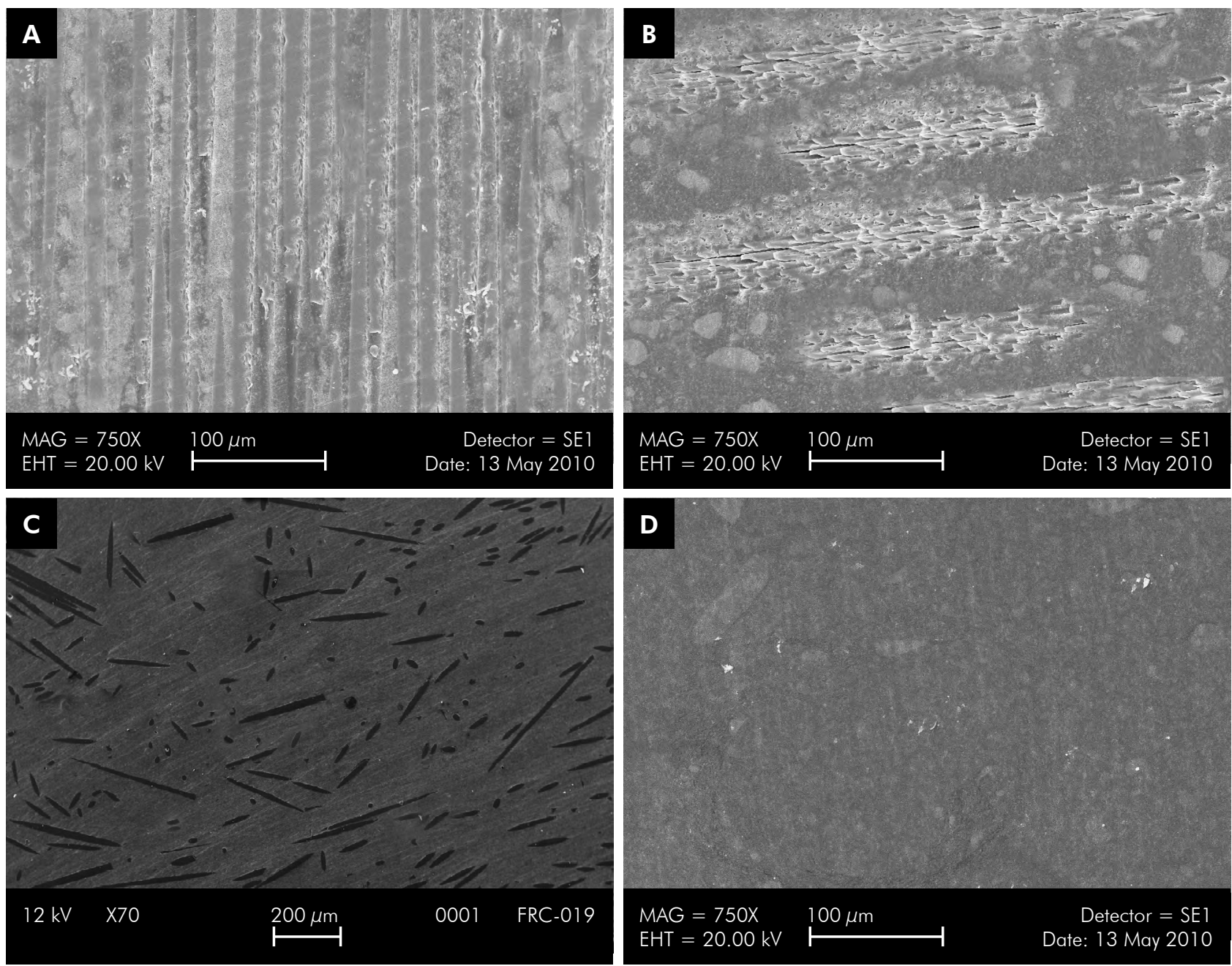

Figure. A. Scanning electron microscopic image of EUFRC, $750 \times$ magnification; B. Scanning electron microscopic image of EBFRC, $750 \times$ magnification; C. Scanning electron microscopic image of ERFRC, $750 \times$ magnification; D. Scanning electron microscopic image of EC, $750 \times$ magnification.

regarding the utility of such restorations in restoration procedures. Recently, FRC fixed partial dentures have been identified as the definitive treatment modality, having an expected survival time of at least 5-6 years, and providing adequate interocclusal space and high patient satisfaction at a low cost. ${ }^{18}$ The fracture resistance of a clinical crown is affected by several factors, including the material, cementation, artificial aging, and loading conditions, as well as the elastic modulus of the supporting die. ${ }^{32}$ Fracture resistance increases with increasing elastic modulus of the supporting material. ${ }^{33}$ However, natural teeth may have lower fracture strength, and it is difficult to standardize the abutments.
In the present study, EUFRC demonstrated the highest load-bearing capacity. The volume fraction affected the load-bearing capacity and crack propagation. High-volume designs have exhibited higher survival rates compared to low-volume designs in previous studies. ${ }^{34}$ However, in our study, ERFRC and EUFRC showed very similar results. Multidirectional reinforcement may reduce strength in a specific direction. Moreover, increased silica filler content contributes to load-bearing capacity. The load-bearing capacity of EBFRC crowns was similar to that of ERFRC and EUFRC. In addition, continuous bidirectional FRCs are stiff and can slow down or attenuate crack propagation. ${ }^{12}$ Accordingly, 
FF values were higher than IF values for FRC crowns. However, fiber reinforcement increased the loadbearing capacity of IF and FF in all groups in our study, as also reported previously. ${ }^{12}$ The CCB samples fractured instantaneously due to the rigidity of the ceramic material, while catastrophic and partial fractures occurred in EC blocks. IF and FF values of the CCB and EC blocks were similar.

FRC restorations can be fabricated manually, but CAD/CAM-controlled manufacturing provides better and more standardized results. Millable composite resin materials have the advantages of less milling bur use, shorter milling time, repairability, and chairside polishing, as well as favoring the wear on antagonist teeth and the lack of any requirement for veneering porcelain. ${ }^{33,35}$ The SEM analysis showed that FRCs and EC resins had less porosity and a higher degree of conversion. The fibers showed a satisfactory ability to penetrate the composite resins; there were no pores between fibers because they were polymerized under pressure in a vacuum chamber. Notably, vacuumimpregnated blocks have high flexural strength. ${ }^{36}$ The FF values in this study suggest that the vacuum method has several advantages.

\section{Conclusion}

Despite the limitations of this study, fiber reinforcement was shown to increase the loadbearing capacity of all specimens tested, in terms of IF and FF. The SEM findings supported our conclusion that composite resins and FRCs can be successfully fabricated by CAD/CAM systems, and could serve as alternatives to posterior crowns.

\section{References}

1. AL-Makramani BM, Razak AA, Abu-Hassan MI. Comparison of the load at fracture of Turkom-Cera to Procera AllCeram and In-Ceram all-ceramic restorations. J Prosthodont. 2009 Aug;18(6):484-8. https://doi.org/10.1111/j.1532-849X.2009.00467.x

2. Quinn JB, Sundar V, Parry EE, Quinn GD. Comparison of edge chipping resistance of PFM and veneered zirconia specimens. Dent Mater. 2010 Jan;26(1):13-20. https://doi.org/10.1016/i.dental.2009.08.005

3. McLean JW, von Fraunhofer JA. The estimation of cement film thickness by an in vivo technique. Br Dent J. 1971 Aug;131(3):107-11. https://doi.org/10.1038/sj.bdj.4802708

4. Leevailoi C, Platt JA, Cochran MA, Moore BK. In vitro study of fracture incidence and compressive fracture load of all-ceramic crowns cemented with resin-modified glass ionomer and other luting agents. J Prosthet Dent. 1998 Dec;80(6):699-707. https://doi.org/10.1016/S0022-3913(98)70058-7

5. Dogon IL. Current status of posterior composite resin in North America. In: Vanherle G, Smith DC, editors. Posterior composite resin dental materials. Boston: Peter Szulc; 1985. p. 163-71.

6. Sarrett DC. Clinical challenges and the relevance of materials testing for posterior composite restorations. Dent Mater. 2005 Jan;21(1):9-20. https://doi.org/10.1016/j.dental.2004.10.001

7. Goldberg AJ, Burstone CJ. The use of continuous fiber reinforcement in dentistry. Dent Mater. 1992 May;8(3):197-202. https://doi.org/10.1016/0109-5641(92)90083-O

8. Nohrström TJ, Vallittu PK, Yli-Urpo A. The effect of placement and quantity of glass fibers on the fracture resistance of interim fixed partial dentures. Int J Prosthodont. 2000 Jan-Feb;13(1):72-8.

9. Bae JM, Kim KN, Hattori M, Hasegawa K, Yoshinari M, Kawada E, et al. The flexural properties of fiber-reinforced composite with light-polymerized polymer matrix. Int J Prosthodont. 2001 Jan-Feb;14(1):33-9.

10. Krejci I, Boretti R, Giezendanner P, Lutz F. Adhesive crowns and fixed partial dentures fabricated of ceromer/FRC: clinical and laboratory procedures. Pract Periodontics Aesthet Dent. 1998 May;10(4):487-98.

11. Krejci I, Boretti R, Giezendanner P, Lutz F. Adhesive crowns and fixed partial dentures of optimized composite resine with glass fiber- bonded framework. Quintetessence Dent Technol. 1999;22:107-27.

12. Dyer SR, Lassila LV, Jokinen M, Vallittu PK. Effect of fiber position and orientation on fracture load of fiber-reinforced composite. Dent Mater. 2004 Dec;20(10):947-55. https://doi.org/10.1016/i.dental.2003.12.003

13. Tezvergil A, Lassila LV, Vallittu PK. The effect of fiber orientation on the polymerization shrinkage strain of fiber-reinforced composites. Dent Mater. 2006 Jul;22(7):610-6. https://doi.org/10.1016/i.dental.2005.05.017 
14. Petersen RC. Discontinuous fiber-reinforced composites above critical length. J Dent Res. 2005 Apr;84(4):365-70. https://doi.org/10.1177/154405910508400414

15. Nielsen LE. Mechanical properties of polymer and composites. New York: M. Dekker; 1974.

16. Cheng TH, Jones FR, Wang D. Effect of fibre conditioning on the interfacial shear strength of glass-fibre composites. Compos Sci Technol. 1993;48(1-4):89-96. https://doi.org/10.1016/0266-3538(93)90123-X

17. Garoushi S, Vallittu PK, Lassila LV. Fracture resistance of short, randomly oriented, glass fiber-reinforced composite premolar crowns. Acta Biomater. 2007 Sep;3(5):779-84. https://doi.org/10.1016/i.actbio.2007.02.007

18. Vallittu PK, Shinya A, Baraba A, Kerr I, Keulemans F, Kreulen C, et al. Fiber-reinforced composites in fixed prosthodontics-Quo vadis? Dent Mater. 2017 Aug;33(8):877-9. https://doi.org/10.1016/i.dental.2017.05.001 PMID:28552334

19. Dyer SR, Lassila LV, Jokinen M, Vallittu PK. Effect of cross-sectional design on the modulus of elasticity and toughness of fiber-reinforced composite materials. J Prosthet Dent. 2005 Sep;94(3):219-26. https://doi.org/10.1016/i.prosdent.2005.06.008

20. Körber KH, Ludwig K. Maximale Kaukraft als berechnungsfaktor zahntechnischer Konstruktionen. Dent Labor (Munch). 1983;31:55-60.

21. Maghrabi AA, Ayad MF, Garcia-Godoy F. Relationship of margin design for fiber-reinforced composite crowns to compressive fracture resistance. J Prosthodont. 2011 Jul;20(5):355-60. https://doi.org/10.1111/j.1532-849X.2011.00713.x

22. Gibbs CH, Anusavice KJ, Young HM, Jones JS, Esquivel-Upshaw JF. Maximum clenching force of patients with moderate loss of posterior tooth support: a pilot study. J Prosthet Dent. 2002 Nov;88(5):498-502. https://doi.org/10.1067/mpr.2002.129062

23. Rinke S, Hüls A, Jahn L. Marginal accuracy and fracture strength of conventional and copy-milled all-ceramic crowns. Int J Prosthodont. 1995 Jul-Aug;8(4):303-10.

24. Gehrt M, Wolfart S, Rafai N, Reich S, Edelhoff D. Clinical results of lithium-disilicate crowns after up to 9 years of service. Clin Oral Investig. 2013 Jan;17(1):275-84. https://doi.org/10.1007/s00784-012-0700-x

25. Behr M, Rosentritt M, Handel G. Fiber-reinforced composite crowns and FPDs: a clinical report. Int J Prosthodont. 2003 May-Jun;16(3):239-43.

26. Beschnidt SM, Strub JR. Evaluation of the marginal accuracy of different all-ceramic crown systems after simulation in the artificial mouth. J Oral Rehabil. 1999 Jul;26(7):582-93. https://doi.org/10.1046/j.1365-2842.1999.00449.x

27. Attia A, Abdelaziz KM, Freitag S, Kern M. Fracture load of composite resin and feldspathic all-ceramic CAD/CAM crowns. J Prosthet Dent. 2006 Feb;95(2):117-23. https://doi.org/10.1016/i.prosdent.2005.11.014

28. Scott R. Dyer, John A. Sorensen, Lippo V. J. Lassila, Pekka K Vallittu. Damage mechanics and load failure of fiber-reinforced composite fixed partial dentures. Dent Mater. 2005;21(12):1104-10. https://doi.org/10.1016/i.dental.2005.03.001

29. Perea-Lowery L, Vallittu PK. Framework design and pontics of fiber-reinforced composite fixed dental prostheses: an overview. J Prosthodont Res. 2018 Jul;62(3):281-6. https://doi.org/10.1016/i.jpor.2018.03.005

30. Gresnigt MM, Özcan M, van den Houten ML, Schipper L, Cune MS. Fracture strength, failure type and Weibull characteristics of lithium disilicate and multiphase resin composite endocrowns under axial and lateral forces. Dent Mater. 2016 May;32(5):607-14. https://doi.org/10.1016/i.dental.2016.01.004

31. Malquarti G, Berruet RG, Bois D. Prosthetic use of carbon fiber-reinforced epoxy resin for esthetic crowns and fixed partial dentures. J Prosthet Dent. 1990 Mar;63(3):251-7. https://doi.org/10.1016/0022-3913(90)90190-N

32. Campbell SD. A comparative strength study of metal ceramic and all-ceramic esthetic materials: modulus of rupture. J Prosthet Dent. 1989 Oct;62(4):476-9. https://doi.org/10.1016/0022-3913(89)90184-4

33. Shearer B, Gough MB, Setchell DJ. Influence of marginal configuration and porcelain addition on the fit of In-Ceram crowns. Biomaterials. 1996 Oct;17(19):1891-5. https://doi.org/10.1016/0142-9612(95)00302-9

34. Freilich MA, Meiers JC, Duncan JP, Eckrote KA, Goldberg AJ. Clinical evaluation of fiber-reinforced fixed bridges. J Am Dent Assoc. 2002 Nov;133(11):1524-34. https://doi.org/10.14219/jada.archive.2002.0084

35. Carvalho AO, Bruzi G, Giannini M, Magne P. Fatigue resistance of CAD/CAM complete crowns with a simplified cementation process. J Prosthet Dent. 2014 Apr;111(4):310-7. https://doi.org/10.1016/i.prosdent.2013.09.020

36. Ruddell DE, Maloney MM, Thompson JY. Effect of novel filler particles on the mechanical and wear properties of dental composites. Dent Mater. 2002 Jan;18(1):72-80. https://doi.org/10.1016/S0109-5641(01)00022-7 